\title{
Application of a New Method of Collecting Oil and Petroleum Products to Improve the Quality of Spill Response
}

\author{
Vitaly A. Onov ${ }^{1 *}$ \\ ${ }^{1}$ Saint - Petersburg University of State Fire Service of EMERCOM of Russia, 196105 St. Petersburg, \\ Russia
}

\begin{abstract}
The article substantiates a new method of localization and elimination of oil and oil products spills. This method is the most environmentally friendly of all existing ones, fast, high-quality, inexpensive, with a low degree of further spread of the spill and a high degree of complete elimination.
\end{abstract}

\section{Introduction}

Currently, the main method of eliminating contaminated oil is layer-by-layer removal of it by bulldozers and excavators in the area of an emergency spill, its transportation and storage to industrial dumps [1]. In the best case, oil-ground is taken to factory or port concreted sites, not limited by sealed shafts from the inevitable runoff into the surrounding soil. For example, on November 11, 2007, the largest disaster in Russia in recent decades occurred in the Kerch Strait. Due to the storm that broke out, four bulk carriers and a tanker sank here. 3 thousand tons of fuel oil, almost 7 thousand tons of technical sulfur poured into the sea. During the liquidation of 3 thousand tons of oil from a self-propelled barge, up to 5 thousand tons of oil and gas were dumped on an extensive berth in the Kerch port. In February, under the hot Crimean sun, the oil-ground hill began to spread intensively along the pier, falling into the sea. Such repeated contamination with oil was quite comparable in damage to the primary one. Moreover, a suitable concrete pit was found at a distance of up to $700 \mathrm{~km}$ at the industrial site of an oil refinery in the Kirovograd region. Additional considerable oil pollution took place along the entire route of transportation of oil and gas by dump trucks with insufficiently sealed bodies.

The following is a typical example of oil spill response in the United States, where, according to experts, advanced technologies and expensive equipment work. During the liquidation of the oil spill in North Dakota, the work took five years and cost more than $\$$ 100 million dollars. U.S. Department of Health scientist Bill Suess reported that more than 699,000 gallons (27,400 tons) were collected - less than $30 \%$ of the spilled oil. The rest turned into oil and gas oil and was removed from the soil by thermal desorption.

\footnotetext{
*Corresponding author: onov.va@igps.ru
} 


\section{Key research findings}

In April 2020, an emergency oil spill occurred in the port of Rotterdam when a tanker collided with a berth. Hundreds of birds were affected due to large-scale water pollution. The main reason is the low efficiency of the existing oil spill response system, namely, the long preparation time for oil recovery vessels, emergency teams of the Netherlands and neighboring countries. Despite large and expensive measures, no more than $40-45 \%$ of the spilled oil was collected.

The procedure for localization and liquidation of emergency oil spills involves a comprehensive solution to the problem, that is, the use of not only various innovative technical means, but also methodological approaches. Regardless of the nature and scale of the accident, first of all, it is necessary to take measures aimed at preventing the spread of oil spills, and then cleaning the contaminated oil ground or water area [2,3].

On land, polluted soil is usually raked by bulldozers and excavated by excavators to the depth of penetration of oil and petroleum products. However, this depth varies greatly in different places depending on the properties of the soil, so it is rarely achieved when emergency spills are eliminated. After selecting the oil-ground, the remaining pit should be evenly filled with sorbents, which will allow the site of an emergency spill to be cleaned as much as possible. The collected masses of oil and gas are transported to industrial dumps and landfills. These masses inevitably spread oil to the depths and around them, creating a new focus of large-scale contamination, which is very difficult to eliminate. Usually these landfills are buried, trying to forget about them.

A lot of money is spent to create biosorbents for ecological oil purification and dispersants for chemical decomposition of oil, but the methods of their supply to spills and especially to contaminate natural substances do not ensure the effective realization of their potential qualities of oil purification. For high-quality cleaning of contaminated soil, it is necessary to place it in layers with sorbent granules in a closed volume. This method is difficult to implement with the help of modern technology, which is unable to efficiently and evenly spray light, brittle sorbent granules over large areas with the effect of penetrating spraying.

Environmental damage and the risk of carcinogenic effects from oil spills are quite comparable to the effects of radioactive dust emissions and larger fragments [4]. The generally accepted technology for storing collected radioactive materials is the construction of single-fill burial grounds, which, in turn, lead to new extensive oil pollution zones [5]. At the same time, there are a number of biological and chemical methods for cleaning materials, but there are no technologies and techniques for evenly mixing biosorbents and dispersants with a mass of soil, sand, mud, algae contaminated with oil. A comprehensive analysis of the above facts allowed us to propose a new method of eliminating the consequences of oil spills of a wide range of scales, including large and catastrophic ones.

The practiced method of soil cleaning is manual, the application of biosorbent with shovels on top of the contaminated soil is low-effective. It can clean a relatively small part of the oil - only a thin surface layer. In fact, more than $90 \%$ of the contaminated soil does not interact with sorbents, but freely expands the contamination zone. They are trying to compensate for this by exporting soil to industrial landfills. They are not designed to store contaminated soil and cannot provide a sufficient degree of isolation from the environment. In addition, this soil is guaranteed to infect all previously loaded industrial waste [6].

Analysis of materials for the elimination of oil spills and petroleum products has shown that the biggest disadvantage of emergency spill response operations is the lack of special and prepared storage sites for contaminated oil and gas. The construction of special tanks for storing contaminated soil is environmentally, economically, socially and technologically justified. 
Contaminated soil differs from radioactive materials in that it can be cleaned of oil using existing biological and chemical technologies, so the tank can be loaded repeatedly [7]. It creates the ideal and most favorable conditions for cleaning oil and mud.

The system solution of the global project is the introduction of an environmentally friendly method of eliminating oil and gas pollution by improving the sorbent spraying process and creating specially designated isolated places for contaminated soil. This will allow us to quickly and efficiently solve the world problem: reduce the degree of environmental pollution in each area of an emergency spill.

A cheap, environmentally friendly and easy-to-implement method of processing oil and gas is proposed, which does not require the creation of new expensive technologies and is based on the use of a combination of ready-made and well-proven technical solutions. It will allow for the first time to put on stream high-quality soil cleaning due to the fact that conditions will be created for the effective realization of the potential of biosorbents and dispersants. Previously, they were discharged unevenly onto the oil film in motion or applied only to the soil surface without mixing, therefore, biosorbents and dispersants showed high efficiency at landfills with uniform penetration into the soil, and in practice with disorderly discharge very low.

For layer-by-layer application of biosorbents to a large mass of soil, it is necessary to create a concrete tank in the form of a swimming pool bath, immersed in the ground, which does not collapse during melting and soil shifts, on the territory of the tank farm of an oil refinery, an oil loading terminal in the port, at a railway station - on a site with convenient entrances and exits. For the convenience of loading and unloading, access platforms and barriers for the dump truck are equipped on the sides of the tank.

After delivery of the contaminated oil-ground to a special tank, the dump truck drives up to the site and simultaneously the chassis with a multi-barrel module (hereinafter MM) (fig. 1) takes a position opposite or to the side of the dump truck (fig. 2, fig. 3). MM creates a vortex covering part of the "bath" under the platform with a layer of sorbents, the dump truck dumps the mass of soil on the biosorbent layer, ensuring continuous contact of the lower surface of the mass with the biosorbent $[8,9]$. Then the MM creates a vortex with a compacted front with a second volley, which carries out penetrating spraying of the sorbent over the upper area of the oil-ground mass. The same three-stage operation is carried out with the second dump truck and each subsequent one:

- creation of the underlying layer of biosorbents by a vortex in a second;

- dumping of the oil-ground mass on it with spreading of the mass over the sorbent layer;

- continuous coating of the upper surface of the mass by the second vortex.

Thus, for the first time in a closed and sealed tank, a multi-layered mass of oil and gas with a biosorbent is created, which ensures the fastest and most effective purification of the soil from oil. The acceleration of the neutralization of the oil component in the "bath" and the absence of pollution of the environment, soil and air is facilitated by the tightness of the tank walls and the presence of a roof with ventilation that prevents the formation of explosive concentrations. 


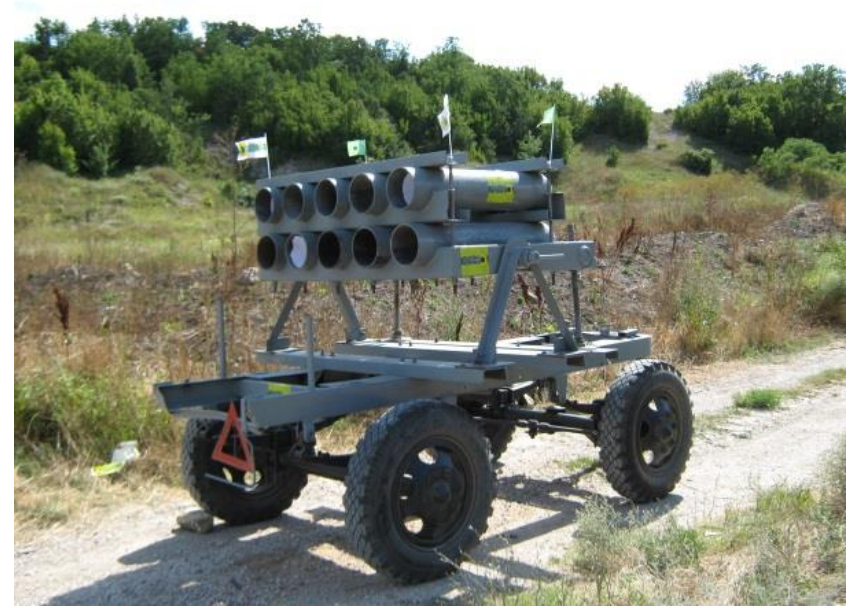

Fig. 1. Multi-barrel module successfully tested to eliminate the consequences of the spill in the Kerch Strait (2007).

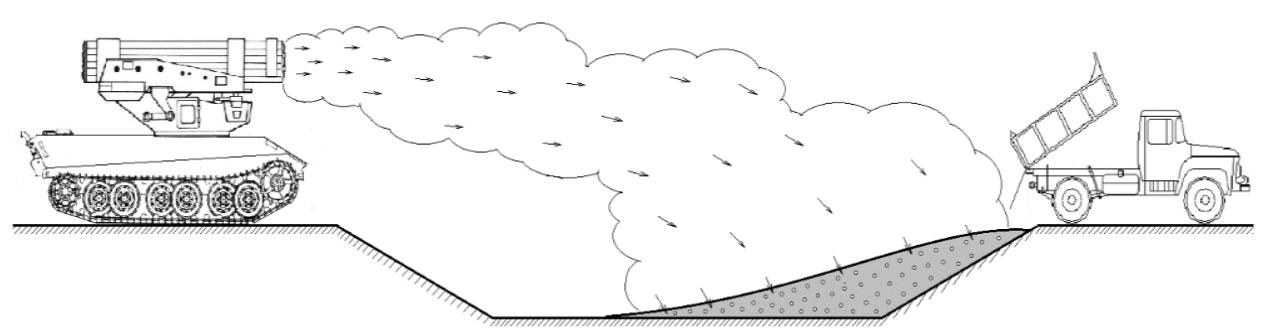

Fig. 2. A dump truck and a machine with MM spraying sorbent in volleys are located at opposite ends of a rectangular tank.

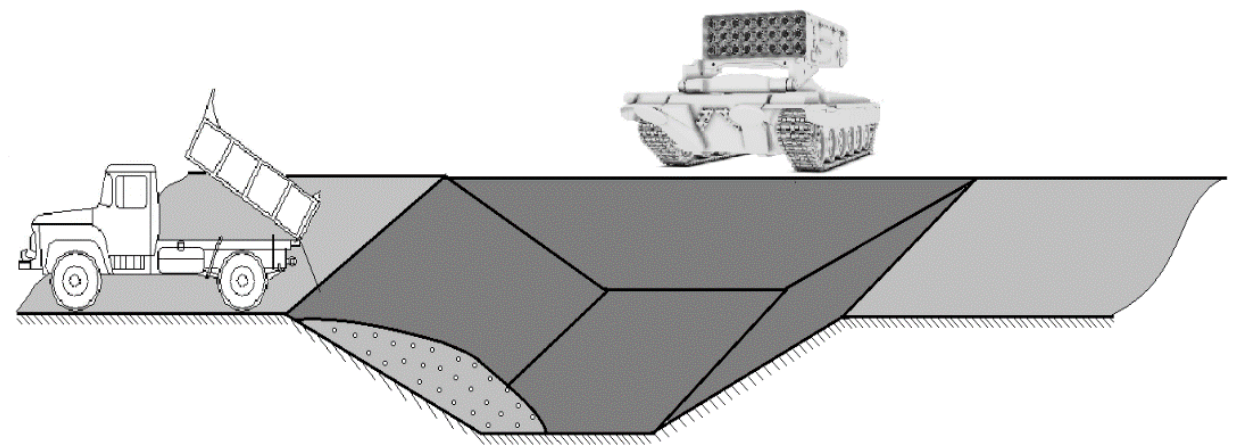

Fig. 3. The dump truck drove up to the side of the tank and dumped the oil-ground, the machine sprayed the sorbent with MM from the end onto the entire outer surface of the mass of the oil-ground.

In remote areas inaccessible or difficult to access for tracked and wheeled landing gear, in case of accidents on pipelines or the spread of an oil spill along rivers, it is advisable to use helicopters with containers of directional spraying or targeted drop initiated at a certain height of 5-20 meters above the surface of the spill [10]. 


\section{Conclusion}

Thus, the introduction of a new method of collecting petroleum products to improve the quality of oil spill response will solve a number of important problems, namely, to avoid large-scale accidents, save costs associated with spill response, and minimize environmental damage.

\section{References}

1. V. I. Pavlenko, Fundamental scientific research in the interests of localization and elimination of oil spills in the Arctic // VESTN. The Security Council of the Russian Federation. - 2011. - № 5 (17). - pp. 154-161.

2. Yu.F. Kaiser, Lysyannikov A.V., Serebrenikova Yu.G., Sherpak E.V., Malianov V.V., Shikharev A.Yu., Esikov V.V., Kudryashov A.F. Organization of localization of oil spill. Collection: Actual problems of introduction of energy-efficient technologies in construction and engineering systems of urban economy. Materials of the II International Scientific and practical conference. 2015. pp. 61-63.

3. Decree of the Government of the Russian Federation, April 15, 2002 "On the Procedure for organizing measures to prevent and eliminate oil and petroleum product spills on the Territory of the Russian Federation".

4. M. G. Gubaidullin, Korobov V. B. Environmental monitoring of oil and gas production facilities in the European North of Russia: A textbook. - Arkhangelsk: CPI North. (Arct.), 2012. p. 236.

5. Techniques and technologies for localization and liquidation of emergency oil and petroleum product spills: Reference/I.A. Meritsidi, V.N. Ivanovsky, A.N. Prokhorov, I.V. Botvinko, I.S. Dubinova, etc. Edited by I.A. Meritsidi. - St. Petersburg, NPO "Professional", 2008. p.824.

6. V.D. Zakhmatov, Prophet V.YA., Kleimenov A.V. Analysis of the development of specialized fire trucks for the protection of oil and gas facilities (part 1) // Problems of risk management in the technosphere, No. 4 (44), 2017. pp. 17-25.

7. A.I. Vylkovan, Ventsyulis L.S., Zaitsev V.M., Filatov V.D. Modern methods and means of combating oil spills: A scientific and practical guide. - St. Petersburg: CenterTechinform, 2000.

8. V.D. Zakhmatov, Tursenev S.A., Chernyshov M.V., Mironchev A.V., Ozerov A.V., Dorozhkin A.S. Analysis of existing and justification of the use of a new automatic system of fire and explosion protection of ships, ships, oil platforms // Fire and Explosion Safety /Fire and Explosion Safety. pp. 50-63. DOI: 18322 / PVB.2018.27.05.

9. Technology of pulse elimination of oil spills at sea, ocean // journal "Successes of modern natural science". pp. 92-99. Awarded a Gold medal at the exhibition of scientific works of the Ministry of Education of Russia, Moscow, 2016.

10. Equipment and technologies for localization and elimination of emergency oil and petroleum product spills: Reference/I.A. Meritsidi, V.N. Ivanovsky, A.N. Prokhorov, I.V. Botvinko, I.S. Dubinova, etc. Edited by I.A. Meritsidi. - St. Petersburg, NGO "Professional", 2008. p. 824. 